\title{
Radiation protection considerations along a radioactive ion beam transport line
}

\author{
Lucia Sarchiapone* and Demetre Zafiropoulos \\ Laboratori Nazionali di Legnaro - INFN \\ Legnaro (Pd), Italy \\ *lucia.sarchiapone@lnl.infn.it
}

Published 1 September 2016

\begin{abstract}
The goal of the SPES project is to produce accelerated radioactive ion beams for Physics studies at "Laboratori Nazionali di Legnaro" (INFN, Italy). This accelerator complex is scheduled to be built by 2016 for an effective operation in 2017. Radioactive species are produced in a uranium carbide target, by the interaction of $200 \mu \mathrm{A}$ of protons at $40 \mathrm{MeV}$. All of the ionized species in the $1+$ state come out of the target (ISOL method), and pass through a Wien filter for a first selection and an HMRS (high mass resolution spectrometer). Then they are transported by an electrostatic beam line toward a charge state breeder (where the $1+$ to $n+$ multi-ionization takes place) before selection and reacceleration at the already existing superconducting linac. The work concerning dose evaluations, activation calculation, and radiation protection constraints related to the transport of the radioactive ion beam (RIB) from the target to the mass separator will be described in this paper. The FLUKA code has been used as tool for those calculations needing Monte Carlo simulations, in particular for the evaluation of the dose rate due to the presence of the radioactive beam in the selection/interaction points. The time evolution of a radionuclide inventory can be computed online with FLUKA for arbitrary irradiation profiles and decay times. The activity evolution is analytically evaluated through the implementation of the Bateman equations. Furthermore, the generation and transport of decay radiation (limited to gamma, beta- and beta+ emissions) is possible, referring to a dedicated database of decay emissions using mostly information obtained from NNDC, sometimes supplemented with other data and checked for consistency. When the use of Monte Carlo simulations was not feasible, the Bateman equations, or possible simplifications, have been used directly.
\end{abstract}

Keywords: Radiation protection; Monte Carlo simulation; radioactivity; ambient dose equivalent.

PACS numbers: 24.10.Lx, 29.38.Gj.

\section{Introduction}

The Studies for the Production of Exotic Species (SPES) project will be hosted at Laboratori Nazionali di Legnaro of INFN. ${ }^{1}$ It is a multipurpose project, involving applications of nuclear physics, production of radionuclides of interest in radiopharmacy, testing of high power targets, cross section determination, and neutron-related research such as single-event-effects. The layout of the SPES project area is shown in Fig. 1.

This is an Open Access article published by World Scientific Publishing Company. It is distributed under the terms of the Creative Commons Attribution 3.0 (CC-BY) License. Further distribution of this work is permitted, provided the original work is properly cited. 


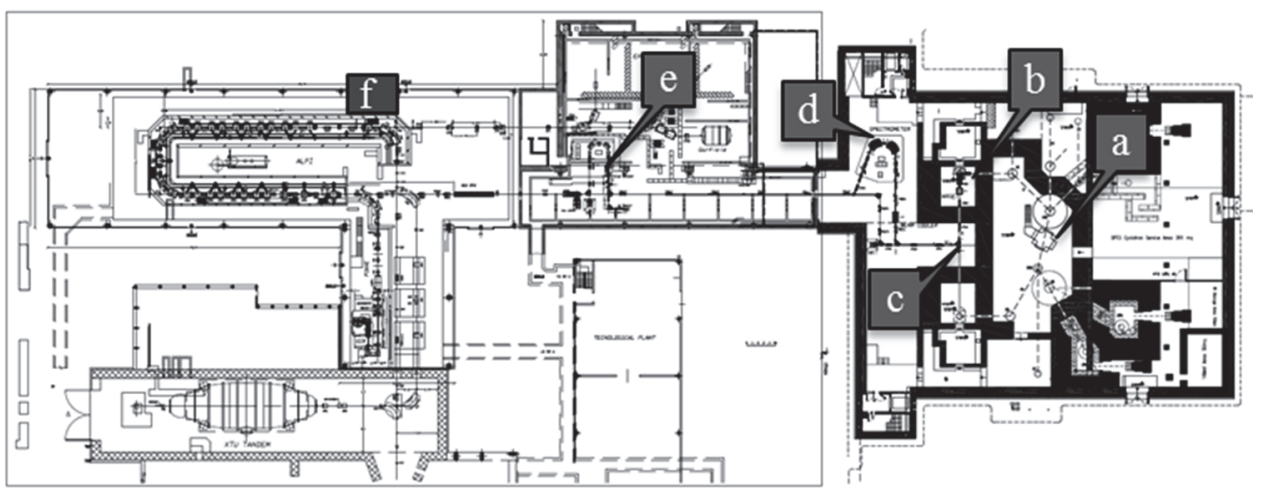

Fig. 1. Layout of the SPES Project area including: a) the cyclotron vault; b) the irradiation bunker hosting the target and the Wien filter; c) the bending magnet; d) the high resolution mass spectrometer; e) the charge breeder; f) the reaccelerating linac ALPI.

The radiation protection service of Laboratori Nazionali di Legnaro will take care of the radiological safety aspects in every stage of the project, starting from the design of the facility up to the dismantling. In particular, the maintenance operations need to be planned. Dedicated areas must be arranged; in case of high dose rates limits to the intervention, times must be set; and personnel equipment that is suitable for the type of operation has to be provided.

In this work, radiation protection considerations are made concerning the dose involved during maintenance of some equipment involved in the nuclear physics application, the generation of radioactive ion beams. In particular, the ${ }^{132} \mathrm{Sn}$ beam has been studied in every selection stage, and results related to this experimental case will be shown.

\section{The Nuclear Physics in the SPES Project}

The nuclear physics program of the SPES project is based on the generation of radioactive ion beams through direct fission of a uranium carbide target induced by a 40 $\mathrm{MeV}$ proton beam with a current of $200 \mu \mathrm{A}$. The p70 cyclotron, a machine produced by Best Cyclotron Inc. and recently installed in the laboratory, will deliver a proton beam with energy of 40 or $70 \mathrm{MeV}$ and current up to $750 \mu \mathrm{A}$.

The radioactive ion beam extracted from the target and ionized at +1 charge state is transported to the Wien filter where a first selection takes place. It is then transported out of the irradiation bunker, bent at $90^{\circ}$, and transferred to the beam cooler and after to the high-resolution mass spectrometer. At this point, the radioactive ion beam is free of any other contaminant beam. Finally, it travels the last piece of the line in +1 charge state towards the charge breeder, where the charge will be enhanced to make the beam acceptable by the linac. 


\subsection{The fissionable target}

The uranium carbide target is being studied and developed at Laboratori Nazionali di Legnaro. It consists of $35 \mathrm{~g}$ of $\mathrm{UC}_{2}$ and is made of 7 disks, $1 \mathrm{~mm}$ thick each, contained in a graphite box inside a tantalum cylinder, heated at $2000{ }^{\circ} \mathrm{C}$ to allow the effusion of the radioactive species. ${ }^{2}$

The released radionuclides migrate to the ionization source where they are ionized in order to be transported by the electrostatic beam line. Depending on the radioactive beam of interest, the ionization will take place through surface, laser or plasma method. The interaction of $40 \mathrm{MeV}$ protons with the target induces $8.2 \times 10^{-3}$ fissions/proton with the fission yield presented in Fig. 2.

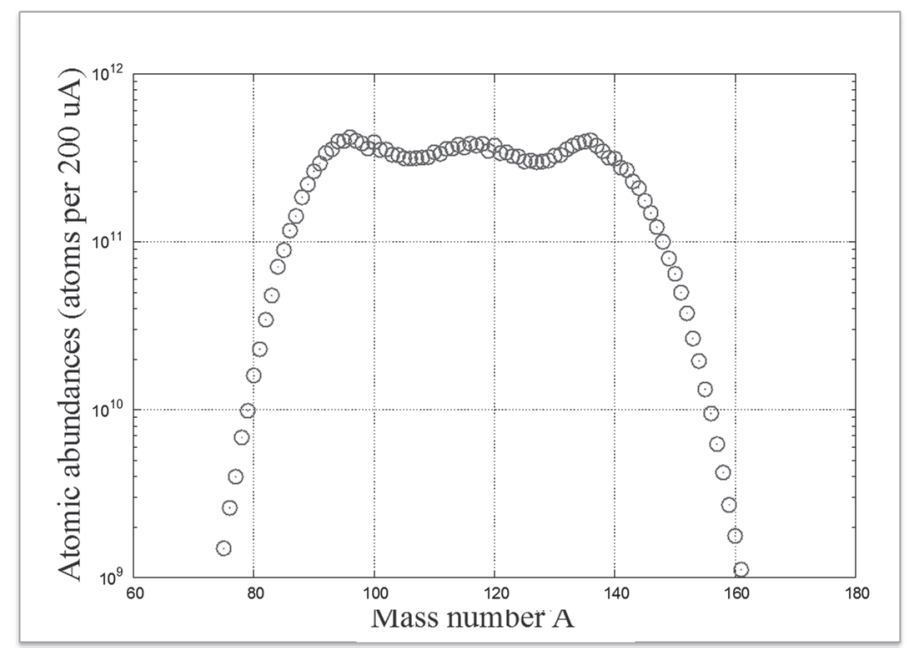

Fig. 2. Fission yield obtained with FLUKA simulation of a proton beam of $40 \mathrm{MeV}$ energy and $200 \mu \mathrm{A}$ current striking a fissionable target.

The total radioactivity of the target at the end of an irradiation cycle lasting 14 days with a proton current of $200 \mu \mathrm{A}$ is $1.2 \mathrm{kCi}$. The secondary neutrons generated by the irradiation of the target induce activation of the materials nearby, in particular the target front end - mainly made of aluminum — and the Wien filter.

\subsection{The Wien filter}

The Wien filter performs the first selection of the radioactive ion beam. The momentum resolution is $1 / 50\left(\Delta p / p\right.$ about $2 \%$ ); for a beam of ${ }^{132} \mathrm{Sn}$, this means that the isobars of mass less than 127 and those of mass higher than 137 will be stopped. The isobars of mass between 127 and 137 will be transported out of the irradiation bunker to the $90^{\circ}$ magnet towards the next resolution steps.

The maintenance of the Wien filter will be done after two years of operation, after removal from the irradiation bunker. The classification of the area where it will be stored 
during maintenance and the organization of the intervention (classification of personnel, time duration, equipment) depend on the radioactivity of the Wien filter, this radioactivity being the sum of two contributions: (1) activation of the constituents of the Wien filter due to the intense neutron field in the bunker; and (2) radionuclides stopped by the Wien filter during the selection of the beam of interest. These radionuclides will build up during several experimental shifts and will act as radioactive sources.

To calculate the first contribution, FLUKA simulations ${ }^{3,4}$ have been run. The possibility to run a simulation with the correct target composition during the irradiation stage and then remove the target (i.e. set the target material "vacuum") during the decay time has been exploited. In this way, the contribution of the fissionable target to the residual dose rate is neglected, and the radioactivity of the other materials can be evaluated.

The second contribution has been calculated considering each radionuclide stopped by the Wien filter, calculating the intensity of the source after two years of operation and build up of that radionuclide, and then summing up the contribution from all the sources to the ambient equivalent dose rate. With maintenance done after some months from the irradiation stop, not all of the radionuclides stopped by the Wien filter have been calculated: only those whose half-life is longer than two weeks (after six months, the radioactivity of a radionuclide with half-life of two weeks is $0.02 \%$ of the initial radioactivity) or whose decay chain includes a nuclide with half-life longer than two weeks are included. In the latter case, the radioactivity of the long-lived daughter is the one under inspection.

For this calculation, instead of running separate simulations for each radionuclide, the full list has been reported in an Excel file, then the selection of nuclides has been done on the basis of the half-life as described above, and finally the contribution to the dose rate from each nuclide has been calculated. When it happened that one of the daughters in a decay chain had a half-life much longer than the parent, the radioactivity of the daughter has been calculated using the parent's current (ions/s) in a simplified Bateman equation.

The ambient equivalent dose rate at $1 \mathrm{~m}$ distance from the Wien filter after one irradiation shift lasting two weeks is $100 \mu \mathrm{Sv} / \mathrm{h}$ (Fig. 3). This is the dose rate due to the activation of the materials of the Wien filter. The dose rate due to the presence of radioactive sources on the Wien filter surface is instead $3 \mathrm{mSv} / \mathrm{h}$ at $1 \mathrm{~m}$ distance after two years of extraction of the ${ }^{132} \mathrm{Sn}$ beam. 


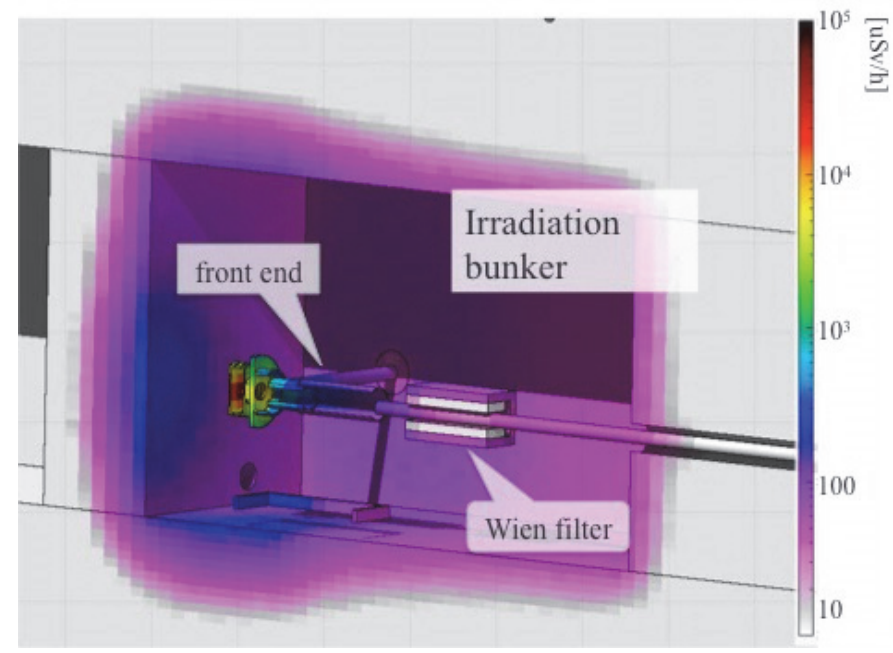

Fig. 3. Graphical representation of the FLUKA simulation results: ambient equivalent dose rate in the irradiation bunker including the target front end and the Wien filter.

\subsection{The $90^{\circ}$ dipole}

The slits intercepting the unresolved beam will be made of copper. They will not need maintenance, but the evaluation of the dose rate at certain distances is still of interest due to the possible presence of personnel after the end of an irradiation cycle. In order to prevent any overexposure in the presence of radioactive sources, the residual dose rate has been calculated for selected beams. In particular for the ${ }^{132} \mathrm{Sn}$ beam selection, the dose rate at one meter from the slits is as high as $2 \mathrm{mSv} / \mathrm{h}$ one week after the end of the irradiation shift.

\subsection{The High Resolution Mass Spectrometer}

As in the case of the $90^{\circ}$ dipole, the unresolved beam will be intercepted by copper slits right after the spectrometer. These elements will not need particular maintenance but the possible presence of personnel after the irradiation cycle is a motivation to evaluate the dose rate at a certain distance. In particular for the ${ }^{132} \mathrm{Sn}$ beam selection, the dose rate at one meter from the slits of the spectrometer is about $250 \mu \mathrm{Sv} / \mathrm{h}$ one week after the end of the irradiation shift.

\subsection{The charge breeder}

The charge state of the radioactive ion beam is enhanced in the charge breeder. ${ }^{5}$ At this stage, the beam of interest is completely resolved. The efficiency of the charge breeder is about $50 \%$ with a fraction of beam lost inside the plasma chamber. Maintenance will be done offline, and eventually the plasma chamber will be removed. 
External exposure may be due to the presence of radioactivity on the internal surface of the plasma chamber. In the presence of volatile radioactivity, there might be inhalation and consequently internal exposure. Evaluations concerning the external exposure have been done with Monte Carlo simulations; the radioactivity of volatile elements involved giving a risk of internal exposure have been done using Bateman equations.

Considering a ${ }^{132} \mathrm{Sn}$ beam of an intensity of $10^{10}$ particles/s, the ambient dose rate ten days after the end of irradiation is higher than $1 \mathrm{mSv} / \mathrm{h}$ in contact with the $\mathrm{CB}$ surface (a graphical representation of the ambient dose rate distribution is given in Fig. 4). Due to the presence of ${ }^{132} \mathrm{I}$, which is volatile, in the ${ }^{132} \mathrm{Sn}$ decay chain, there is a risk of internal exposure if the ${ }^{132} \mathrm{I}$ is inhaled.

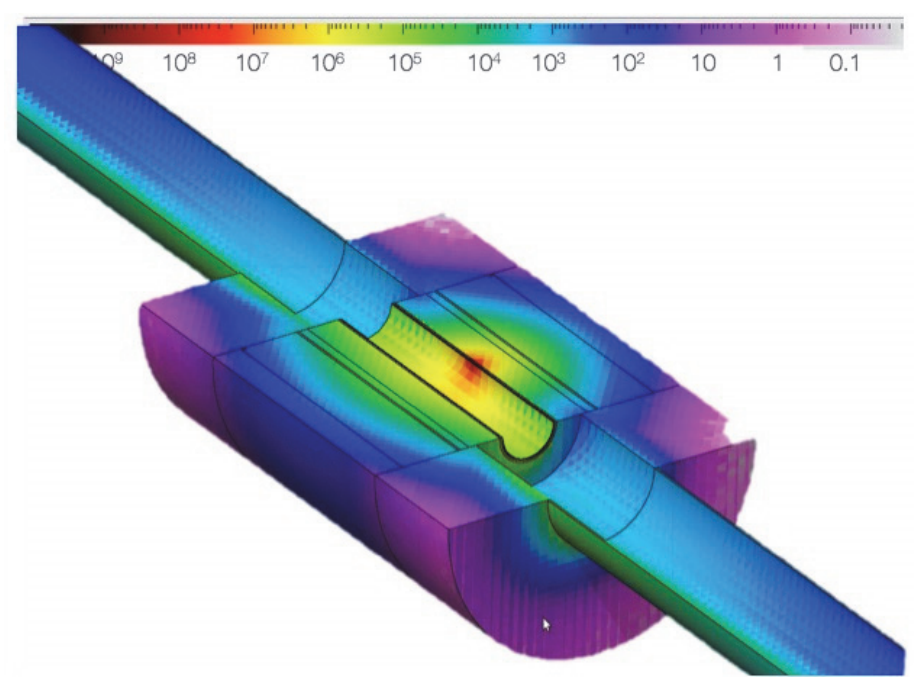

Fig. 4. Schematic section of the charge breeder as included in the FLUKA geometry, mapped with results of ambient dose equivalent calculations $(\mu \mathrm{Sv} / \mathrm{h})$.

The operation of the charge breeder with radioactive ion beams other than ${ }^{132} \mathrm{Sn}$ might not be as troublesome. For ${ }^{90} \mathrm{Rb},{ }^{94} \mathrm{Kr}$ and ${ }^{138} \mathrm{Xe}$ beams, the ambient dose rate in contact with the object is less than $1 \mu \mathrm{Sv} / \mathrm{h}$ one week after the end of the irradiation. For beams of ${ }^{135} \mathrm{I},{ }^{137} \mathrm{Te}$ and ${ }^{134} \mathrm{Sn}$, the ambient dose rate in contact with the object is about $0.1 \mathrm{mSv} / \mathrm{h}$ after one week. A reasonable cooling time of approximately two weeks after the end of the irradiation reduces the risk of internal exposure for all of these beams.

\section{Conclusion}

The generation of a radioactive ion beam in the SPES project is realized through several steps for the selection of a "clean" beam, starting from fission products. Each instrument on the beam line will intercept a fraction of the unresolved beam and will be 
contaminated by the radioactive nuclides sticking on the surfaces. The dose rate due to the presence of these radioactive sources has been calculated, especially in the case of ${ }^{132} \mathrm{Sn}$ production.

It has been seen that whenever maintenance on an instrument has to be done offline, dedicated spaces classified as "controlled area" must be set, in particular for the charge breeder. When maintenance is not urgent and when the number of sources contributing to the dose rate is high, as it is for the Wien filter, it is better to wait before handling the object (a reasonable time might be six months to allow species with half-life of the order of two weeks to decay). In case volatile radionuclides are present, personnel must operate wearing suitable equipment to avoid the risk of radioactivity inhalation.

\section{References}

1. G. Prete and A. Covello, SPES Technical Design Report, April 2008, INFN-LNL-223.

2. A. Andrighetto, C.M. Antonucci, S. Cevolani, C. Petrovich, and M. Santana Leitner, Eur. Phys. J. A30, 591 (2006).

3. A. Ferrari, P.R. Sala, A. Fasso` and J. Ranft, FLUKA: a multi-particle transport code, CERN 2005-10 (2005), INFN/TC_05/11, SLAC-R-773.

4. T.T. Böhlen, F. Cerutti, M.P.W. Chin, A. Fassò, A. Ferrari, P.G. Ortega, A. Mairani, P.R. Sala, G. Smirnov and V. Vlachoudis, Nuclear Data Sheets 120, 211-214 (2014).

5. A. Galatà, G. Patti, C. Roncolato, J. Angot and T. Lamy, Review Sci. Instr. 87, $02 B 503$ (2016); doi: $10.1063 / 1.4933338$. 